\title{
FIELD MEASUREMENTS OF THE KEK B-FACTORY DIPOLE AND WIGGLER MAGNETS
}

\author{
K. Egawa $^{\#}$, M. Masuzawa, KEK, Tsukuba, Japan
}

\section{Abstract}

The KEK B-Factory (KEKB) [1] consists of $3.5 \mathrm{GeV}$ positron (LER) and $8.0 \mathrm{GeV}$ electron (HER) rings and has more than 290 dipole and 150 wiggler magnets. Both the LER and the HER use four types of dipole magnets. Wiggler magnets are used for the LER. Three long flipflop coil systems were used to measure the transverse distribution of the integrated magnetic field along the beam direction. Each dipole magnet was measured using one of these system according to its size. The wiggler magnets were measured using a rotating coil which contains one long coil and two half coils. The long coil measured the net residual integrated magnetic field and each half coil measured the magnetic flux of each pole. Field mapping has also been performed for at least one of each type of magnet. Effective length and field distribution along the beam direction were measured by using a flipflop mapping coil system. The absolute magnetic field strength at dipole center was calibrated by NMR. These measurement results are summarized.

\section{MAGNET SYSTEM}

Many kinds of magnets more than 1600 magnets are used for the KEKB besides correctors. Quarter of the main magnets are recycled from the TRISTAN electron-positron collider (TR) [2]. All the magnets were newly fabricated for the LER. All the dipoles except one are recycled for the HER. The main parameters of the dipole and wiggler magnets are summarized in Table 1. All the cores except $\mathrm{B}_{\mathrm{V}}$ and $\mathrm{B}_{\mathrm{S}}$ are made by using laminated construction technique. The required magnetic properties of the steel are shown in Table 2. The main coil is made of $\mathrm{O}_{2}$ free $\mathrm{Cu}$ hollow conductor. Each magnet has correction coil with about $1 \%$ ampere-turns compared to the main coil and also has 1 turn coil per pole for magnetic flux monitor. The brief summary of the KEKB magnet system is given in the contribution of EPAC98 [3].

Table 2: Magnetic Properties of the silicon steel

\begin{tabular}{|ll|}
\hline $\mathrm{B}_{50}>1.6 \mathrm{~T}$ & $\Delta \mathrm{B}_{50} / \mathrm{B}_{50}< \pm 1 \%$ \\
$\mathrm{Hc}^{1.5}<70 \mathrm{~A} / \mathrm{m}$ & $\Delta \mathrm{Hc}^{1.5} / \mathrm{Hc}^{1.5}< \pm 5 \%$ \\
\hline
\end{tabular}

\section{MEASUREMENTS}

The tolerances of higher multipole errors required for the dipole magnets are given in Table 3. Three flip coils and one rotating coil were used for the measurement of the dipole and the wiggler magnets respectively. The effective length and field distribution along the beam direction were measured by using a flip-flop mapping coil.

Table 3: Tolerances of systematic multipole errors

\begin{tabular}{|l|l|}
\hline & Tolerance at 50 mm radius \\
\hline Dipole magnets & B3 / B1 $<0.12 \%$ \\
& B5 / B1 $<0.45 \%$ \\
\hline
\end{tabular}

\subsection{Series Measurement}

All the dipoles except $\mathrm{B}_{\mathrm{V}}$ were measured by the flip coil systems. The LER $B_{\text {arc }}$ were measured by a newly constructed $2 \mathrm{~m}$ flip coil. The LER $\mathrm{B}_{\mathrm{lc}}$ for chromaticity correction and the HER $B_{\text {arc }}$ were measured by $3.6 \mathrm{~m}$ and $6.6 \mathrm{~m}$ flip coils recycled from TR respectively. Each probe has a few turns coil wound on glass epoxy cylinder. At each system, a pair of test and reference dipoles are facing each other and the probe moves horizontally between two dipoles. The integrated magnetic field $\mathrm{BL}$ (integrated along beam direction) was obtained to measure the induced voltage by flipping the probe in the dipole field. MetroLab high precision integrators (PDI5025) were used to digitize the induced voltage. The BL's were measured along transverse direction at several different currents. After measuring a test magnet, the probe came back to the reference magnet and measured it to eliminate

Table 1: LER and HER Dipole Magnet Parameters

\begin{tabular}{|c|ccccccc|}
\hline & $\mathrm{g} / 2(\mathrm{~mm})$ & L.lam $(\mathrm{m})$ & $\mathrm{B}(\mathrm{T})$. dsgn & No.of.mag & ampere/pole & L.eff $[\mathrm{m}]$ & $\mathrm{B}[\mathrm{T}] . \mathrm{meas}$ \\
\hline LER / Barc & 57 & 0.76 & 0.848 & 134 & $1250 \mathrm{~A} \times 32$ & 0.8876 & 0.83616 \\
Blc & 57 & 2.1 & 0.52 & 26 & $1000 \mathrm{~A} \times 24$ & 2.2273 & 0.52697 \\
Bv & 55 & 1.2 & 0.2 & 4 & $500 \mathrm{~A} \times 18$ & 1.3561 & 0.20523 \\
Bs & 57 & 0.3 & 0.21 & 3 & $500 \mathrm{~A} \times 20$ & 0.4085 & 0.21994 \\
wigg & 55 & 0.75 & 0.77 & 192 & $944.4 \mathrm{~A} \times 36$ & 0.6939 & 0.77056 \\
HER / Barc & 35 & 5.804 & 0.3 & 117 & $840 \mathrm{~A} \times 10$ & 5.9006 & 0.29974 \\
Bw & 35 & 2.8 & 0.048 & 6 & $10 \mathrm{~A} \times 150$ & & \\
BsFL & 35 & 1.14 & 0.214 & 1 & $500 \mathrm{~A} \times 12$ & 1.2393 & 0.21473 \\
BsFR & 57 & 0.76 & 0.339 & 1 & $500 \mathrm{~A} \times 32$ & 0.8935 & 0.35205 \\
\hline
\end{tabular}

\# Email : kazumi.egawa@kek.jp 
system errors. The typical measurement results of LER $\mathrm{B}_{\text {arc }}$, HER $\mathrm{B}_{\text {arc }}$ and LER $\mathrm{B}_{\mathrm{lc}}$ are shown in Fig.1, 2 and 3 respectively. The BL's of the LER Barc's were adjusted by the end shim considering the fabrication error of the core length which was thought to be worse than the longer dipoles due to its short length. The result is shown in Fig. 1 lower left. The measurement accuracy of relative $\mathrm{BL}$ is about a few $\times 10^{-5}$ as shown in Fig. 1 lower right.
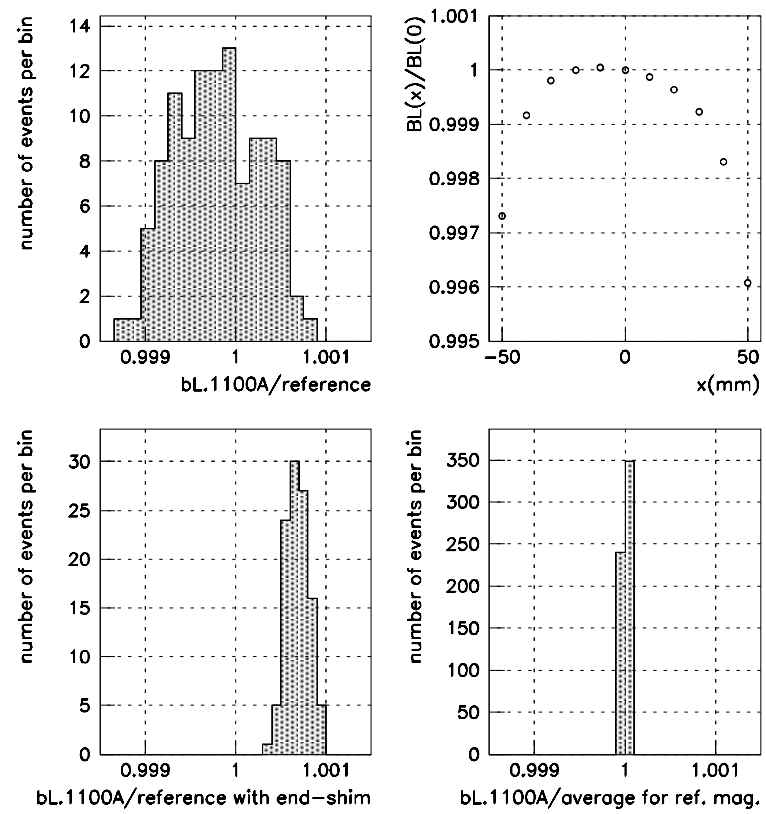

Figure 1 : The normalized integrated field histogram and an example of its transverse distribution for LER $B_{\text {arc }}$. The BL's after end shim correction and the reproducibility of the LER flip coil system are also shown below.
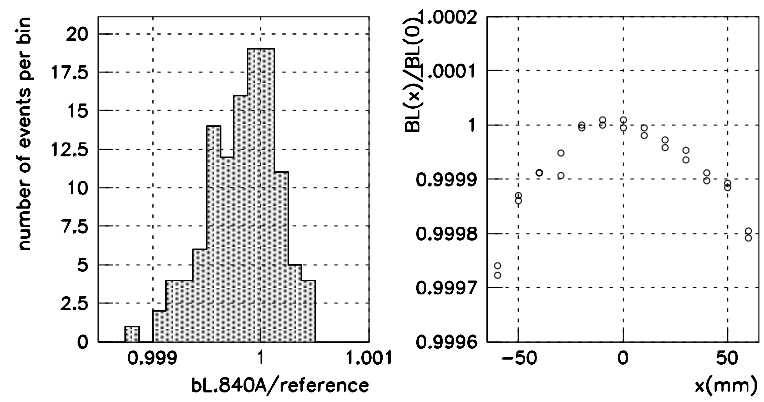

Figure 2 : The normalized integrated field histogram and an example of its transverse distribution for HER $\mathrm{B}_{\text {arc }}$
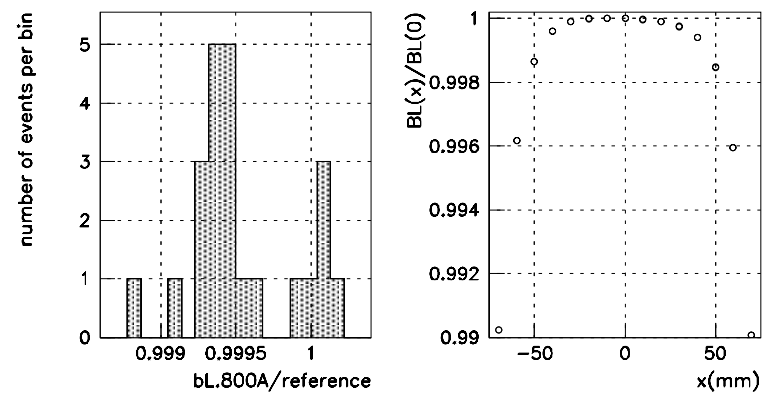

Figure 3 : The normalized integrated field histogram and an example of its transverse distribution for LER Blc
The LER wigglers have alternate magnetic field in their front and back poles. They were measured by the rotating coil mounted in aluminum cylinder having one long coil and two half coils. The residual integrated field $\Delta[\mathrm{BL}]$ was measured by the long coil and the BL for each pole was obtained by each half coil. The induced voltage of the probe was measured by using a spectrum signal analyzer HP3562A. The results are shown in Fig. 4. The little non-zero offset of $\Delta[\mathrm{BL}]$ is thought to be due to the configuration of the current leads.
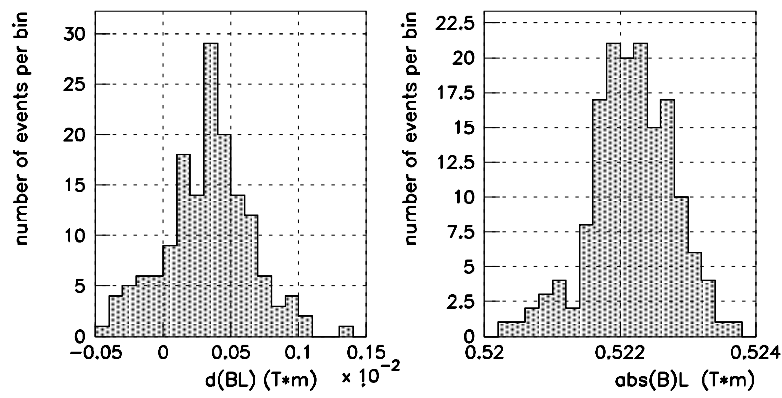

Figure $4:$ The $\Delta[\mathrm{BL}]$ and $|\mathrm{B}| \mathrm{L}$ histograms of the wigglers at $\mathrm{I}=950 \mathrm{~A}$

All the dipoles except $\mathrm{Bw}$ have correction coils. Once the correction coil is excited, the magnet changes the field from its initial value even if the correction current is set to be $0 \mathrm{~A}$ again. These hysteresis effects were measured on the every dipoles and some results are shown in Fig. 5.
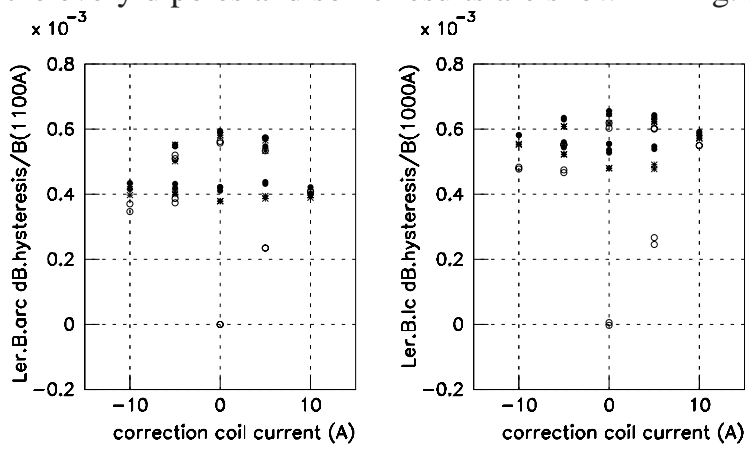

Figure 5 : The hysteresis caused by the correction coil excitation for LER $\mathrm{B}_{\text {arc }}$ and LER B $\mathrm{B}_{\mathrm{lc}}$ at $\mathrm{I}=1100 \mathrm{~A}$ and $1000 \mathrm{~A}$ respectively. The $\mathrm{dB}$ is defined as $\mathrm{dB}[\mathrm{Ic}]=\mathrm{B}[\mathrm{Ic}]-$ $\mathrm{k} * \mathrm{Ic}-\mathrm{B}[\mathrm{Ic}=0 \mathrm{~A}]$, where Ic is the correction current and $\mathrm{k}$ is a linear fit coefficient of B[Ic] as a function of Ic. The white circles show dB's in the first $\pm 10 \mathrm{~A}$ loop, the asterisks and the black circles are in the second and the third loops respectively.

\subsection{Field Mapping and Absolute Value}

The field mappings have been performed by using a flipflop mapping coil, which consists of twin small coils apart from 10mm each other. Each coil has 2090 turns windings with $8 \mathrm{~mm}$ in diameter and $12 \mathrm{~mm}$ height. Dipole field was measured by using one coil. On the other hand, quadrupole field was obtained by measuring the difference of the induced voltages of two coils (twin mode). Each coil gain was calibrated in the dipole field which absolute strength was determined by NMR. The distance between each coil was determined by searching 
zero-cross of the induced voltage of each coil in quadrupole field. The field mappings were carried out at several currents for each type of dipole and wiggler. The effective lengths were determined by these field mappings. The absolute field strength of each type of dipole was measured at its center by NMR. The effective length of each type quadrupole was measured by the twin mode field mapping. The absolute field gradient of the quadrupoles were calculated from the gain and the distance of the twin coils. Some results of the field distributions along the beam direction are shown in Fig. 6, 7, 9 and 10. The effective lengths are shown as functions of the currents for LER $\mathrm{B}_{\mathrm{arc}}$ and LER $\mathrm{B}_{\mathrm{lc}}$ in Fig. 8. This flip-flop mapping coil were also used to measure the magnetic couplings between adjacent quadrupole and corrector. The quadrupole mapping results [4] and the coupling effects [5] are mentioned in the other contributions of this conference.
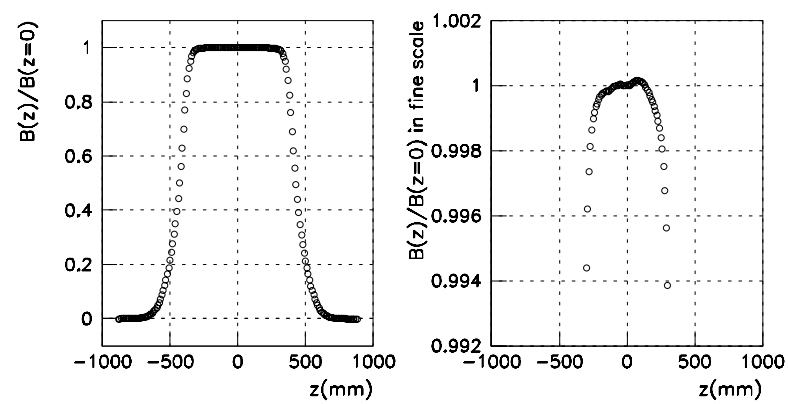

Figure 6: The normalized magnetic field along the beam direction $b[z]$ for LER $B_{\text {arc }}$.
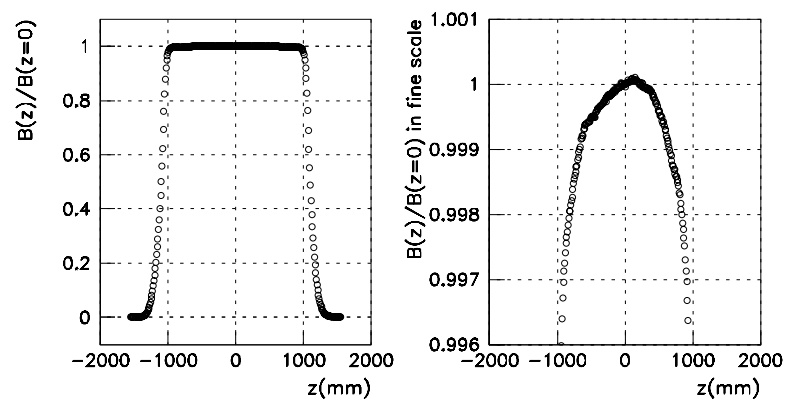

Figure 7 : The $b[z]$ for LER Blc.
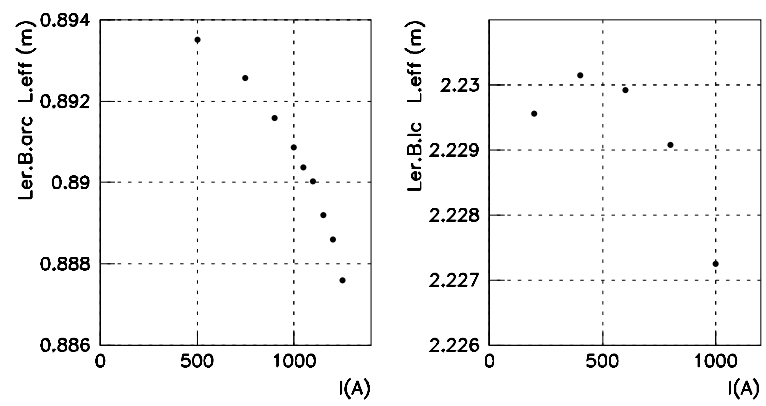

Figure 8: The effective lengths as a function of current for LER B $\mathrm{arc}_{\text {and } \mathrm{LER} B \mathrm{~B} \text {. }}$.
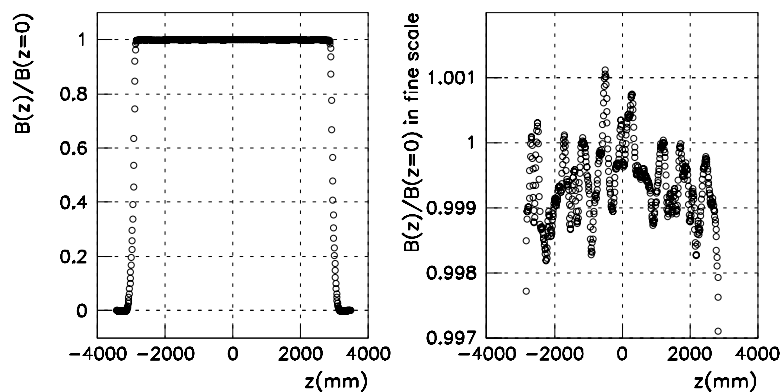

Figure 9: The $b[z]$ for HER $B_{\text {arc }}$.
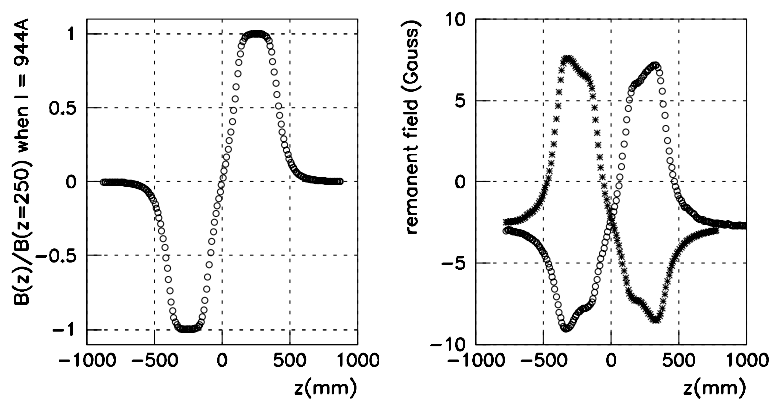

Figure 10: The $b[z]$ at $I=944 A$ (left) and the remanent field along the beam direction (right) for LER Wiggler. In the right figure, the white circle shows the remanent field after +944A excitation and the asterisk shows the remanent after -944A excitation. The negative offset is thought to be due to the terrestrial magnetic effect.

\section{SUMMARY}

The series measurements and the field mappings of the KEKB magnet system were completed. The performances of the magnets are almost acceptable. The measured parameters such as the BL, B'L, effective lengths and magnetic coupling effects are utilized in the operation of the KEKB. Further measurements of environmental effects (air temperature, cooling water flow and its temperature, etc.) on magnetic field are under way.

\section{ACKNOWLEDGMENTS}

The authors would like to thank the measurement crew for their hard work and continuous efforts and also thank the KEK magnet group for many useful discussions and their help.

\section{REFERENCES}

[1] KEKB B-Factory Design Report 95-7

[2] TRISTAN electron-positron colliding beam project", KEK Report 86-24 March 1987

[3] K. Egawa and M. Masuzawa, "Magnet field measurement for KEK B-Factory”, EPAC'98, Stockholm (1998)

[4] M. Masuzawa and K. Egawa, "Field Measurement Results of The KEK B-Factory Quadrupole and Sextupole Magnets", Contribution to PAC99

[5] M. Masuzawa and K. Egawa, "Study of The Magnetic Coupling between Quadrupole and Corrector Magnets for The KEK BFactory", Contribution to PAC99 